Aus dem Institut für Nutztierwissenschaften der Humboldt-Universităt zu Berlin

UWE MÜLLER, WOLFGANG LEUCHT, PETER REINECKE Und TORSTEN DALLE

\title{
Züchtungsbiologische Bewertung der Leistungsselektion beim Milchrind
}

\author{
Herrn Professor Dr. Dr. h.c. Gottfried Leuthold zum 65. Geburtstag gewidmet
}

\begin{abstract}
Summary
Title of the paper: Breeding and biological evaluation of yield selection in dairy cattle

In the course of natural selection metabolic pathways has been improved with regard to reproductive fitness. Yield selection, however, aimes at specific alterations in favour of other traits. The directed reorganization of the genotypic and metabolic constellation leads to an increasing number of individuals, disturbed in their biological balance. This fact has been proved on the example of milk selection in cattle.
\end{abstract}

Key words: dairy cattle, fitness, yield, selction, genetic antagonism, health, fertility, metabolism

\section{Zusammenfassung}

Im Verlaufe der natürlichen Selektion wurden Stoffwechselabfolgen hinsichtlich der reproduktiven Fitneß optimiert. Leistungsselektion zielt aber auf spezifische Veränderungen zu Gunsten anderer Merkmale ab. Die gerichtete Neuordnung der genotypischen und damit auch der stoffwechselphysiologischen Konstellation kann zu einer Häufung von Individuen mit gestörter biologischer Balance führen. Dies konnte im vorliegenden Fall am Beispiel der Milchmengenselektion beim Rind nachgewiesen werden.

Schlïsselwörter: Milchrind, Fitneß, Leistung, Selektion, genetische Antagonismen, Tiergesundheit, Fertilität, Stoffwechsel

\section{Einleitung}

Vor 20 Jahren berichtete PIRCHNER (1979) über Merkmalsantagonismen beim Rind und stellte fest, daß es für die Existenz antagonistischer Beziehungen zwischen Milchleistung und Fruchtbarkeit noch keinen wissenschaftlich fundierten Beweis gibt, sehr wohl aber im Falle anderer Merkmale. Ziel der vorliegenden Arbeit soll es sein, auf der Grundlage neuerer Literaturbefunde und vor dem Hintergrund eines konsequent weiterentwickelten Leistungsniveaus, die Situation beim Milchrind erneut zu analysieren, wobei neben den genetischen auch physiologische Zusammenhänge aufgegriffen und diskutiert werden.

\section{Grundzüge der Selektion}

$2.1 \quad$ Biochemisch-physiologische Veränderungen

Jede genetische Veränderung von Merkmalen beruht auf Veränderungen am genetischen Programm für Stoffwechselabfolgen. Dies erklärt, weshalb Hormonspiegel, En- 
zymaktivitäten, Metabolitenkonzentrationen und andere biochemisch-physiologische Kriterien häufig charakteristische rassen- oder leistungsspezifische Unterschiede aufweisen (BLUM und KUNZ, 1980; WOOLLIASMS und LOVENDAHL, 1991; MIN et al., 1993; MÜLLER et al., 1997).

Da der Stoffwechsel ein kompliziertes Netzwerk aus vielen, sich z.T. selbst oder gegenseitig regulierenden Reaktionen und Teilprozessen ist, wirken nahezu alle genetischen Veränderungen gleichzeitig auf mehrere Merkmale. Solche, als pleiotrope Genwirkungen bezeichneten Effekte sind eine Ursache für genetische Korrelationen. Eine andere Ursache verbirgt sich hinter der gekoppelten Weitergabe von Allelen auf eng benachbarten Genloci, wodurch die Informationen für mehrere Stoffwechselabfolgen im Verbund vererbt werden. Von züchterischer Bedeutung ist, daß pleiotrope Beziehungen nach dem Erreichen eines bestimmten genetischen Niveaus mehr oder weniger dynamischen Veränderungen unterliegen können. So lassen sich z.B. zwei Stoffwechselwege, welche das gleiche Substrat verbrauchen, aber unterschiedliche Merkmale unterstützen, nicht beliebig linear weiterentwickeln. Wenn die Kapazität des Metabolitenpools erschöpft ist, werden sie zu konkurrierenden Teilsystemen. Dann wird dasjenige von beiden die oberste Priorität erhalten, welches in der konkreten Situation zum Erreichen des Selektionszieles am wichtigsten ist.

\subsection{Biochemisch-physiologische Merkmale als indirekte Selektionskriterien}

Die kausalen Zusammenhänge zwischen genotypischer Konstellation, biochemischphysiologischen Kriterien und züchterisch interessierenden Merkmalen legen den Gedanken nahe, einige dieser Kriterien für die indirekte genetische Verbesserung von speziellen Leistungen oder schwer zugänglichen Merkmalen zu nutzen. Mit den methodischen Möglichkeiten und Grenzen solcher Verfahren setzte sich bereits LEUTHOLD (1972) ausführlich auseinander.

Prinzipiell gilt, daß die Konzentrationen und Aktivitäten biochemisch-physiologischer Kennwerte von vielen Genen und nichtgenetischen Faktoren beeinflußt werden. Daher sind sie den quantitativen Merkmalen zuzuordnen und werden nach den gleichen Gesetzmäßigkeiten vererbt wie die meisten Leistungskriterien.

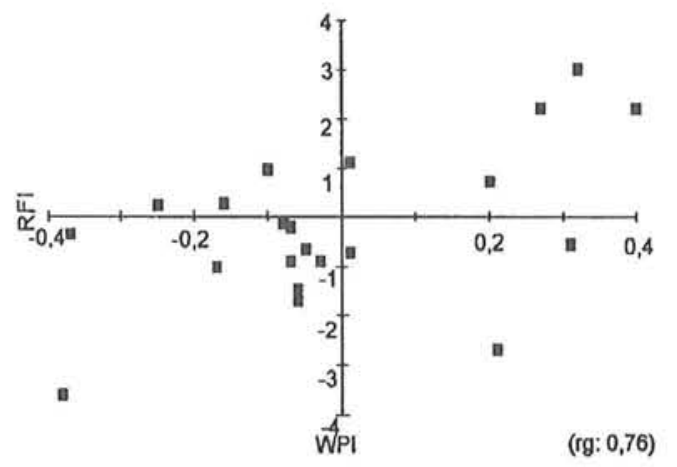

Abb. 1: Beziehungen zwischen den Zuchtwerten für einen Wertepoolindex (WPI) und der residual feed intake (RFI) (Relationships between breeding values for a pooled index and residual feed intake (RFI)) 
Aus Untersuchungen an Milchkühen (s. Abb. 1, MÜLLER et al., 1995) und Milchrindbullen (s. Abb. 2, MÜLLER et al., 1997) geht hervor, daß die aus mehreren Kennwerten zusammengesetzten physiologischen Indizes in ihrer Funktion als indirekte Selektionskriterien häufig größere Zuchtfortschritte erwarten lassen als einzelne Kennwerte und die Gefahr für unbalancierte Stoffwechselveränderungen herabsetzen.

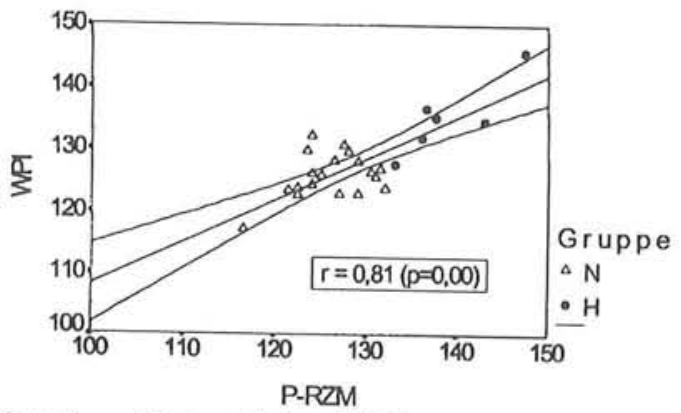

Abb. 2: Beziehungen zwischen einem Wertepoolindex (WPI) und dem Pedigreezuchtwert für Milch (P-RZM) bei Wartebullen (Relationships between a pooled index (WPI) and pedigree breeding value for milk (P-RZM) in dairy bulls)

Tiere der H-Gruppe besaßen höhere P-RZM- Werte als Tiere der N-Gruppe.

\section{$2.3 \quad$ Unterschiede zwischen natürlicher und künstlicher Selektion}

Das Kriterium der natürlichen Selektion ist die reproduktive Fitneß. Man versteht darunter den Beitrag, welchen ein Genotyp für die nachfolgende Generation leistet. Dieser Beitrag wird um so größer sein, je mehr fortpflanzungsfähige Nachkommen ein Individuum Zeit seines Lebens hervorbringt. Hieraus ergibt sich, daß Lebensdauer und Fertilität zwei tragende Säulen der reproduktiven Fitne $\beta$ sind. Alle Spezies sind so programmiert, daß beides, Lebensdauer und Fertilität, sowohl spezifische Optima als auch Grenzen besitzen. Dies ist nicht nur für den Erhalt der genetischen Variabilität in einer Population von Bedeutung, sondern auch für den des sie umgebenden natürlichen Gefüges. Die höchste Fitneß besitzen häufig Individuen im intermediären Optimum, welche in allen Einzelmerkmalen (z.B. Kraft, Schnelligkeit, Resistenz, Reproduktion) eine etwa durchschnittliche Merkmalsausprägung aufweisen.

Die künstliche Selektion zielt auf die Etablierung von Allelen ab, welche in ihrer Gesamtheit einen positiven Einfluß auf bestimmte Teile des Stoffwechsels ausüben. Dies bedeutet aber auch, daß die unter natürlichen Bedingungen entstandene genotypische Konstellation verändert wird und daß Allele mit positivem Einfluß auf die Fitne $\beta$, aber nachteiligen Auswirkungen auf das Selektionsmerkmal allmählich eliminiert werden. Daher wurden bisher in fast allen Selektionsexperimenten unerwünschte Nebeneffekte hinsichtlich Fitneß festgestellt (FALCONER, 1984). Wie, wann und in welchem Aus$\mathrm{ma} ß$ sie sich äußern, hängt u.a. vom Selektionsmerkmal selbst, dem Stadium der Selektion und den jeweiligen Umweltbedingungen ab, welche auf die Population einwirken. Sofern ein bestimmter kritischer Punkt nicht überschritten wird, sind derartige Veränderungen aber immer reversibel. Ermöglicht werden könnte dies z.B. durch genetisch manifestierte Reserve- und Hilfswege im Stoffwechsel, welche von der Selek- 
tion zunächst weitgehend unbeeinflußt bleiben. Sie erlangen erst unter veränderten physiologischen Anforderungen an Bedeutung. Eine weitere Möglichkeit ergibt sich, wenn die nachteilige Entwicklung in einzelnen Fitneßkomponenten durch vorteilhafte Veränderungen in anderen Komponenten zeitweise kompensiert wird (z.B. Lebensdauer durch Fertilität oder umgekehrt).

3. Leistungsselektion beim Milchrind

Zwischen 1970 und 1996 betrug der Anstieg der durchschnittlichen Milchmengenleistung pro Kuh und Jahr beim Schwarzbunten Rind (MLP-Kühe, alte Bundesländer) etwa $50 \%$ (Abb. 3).

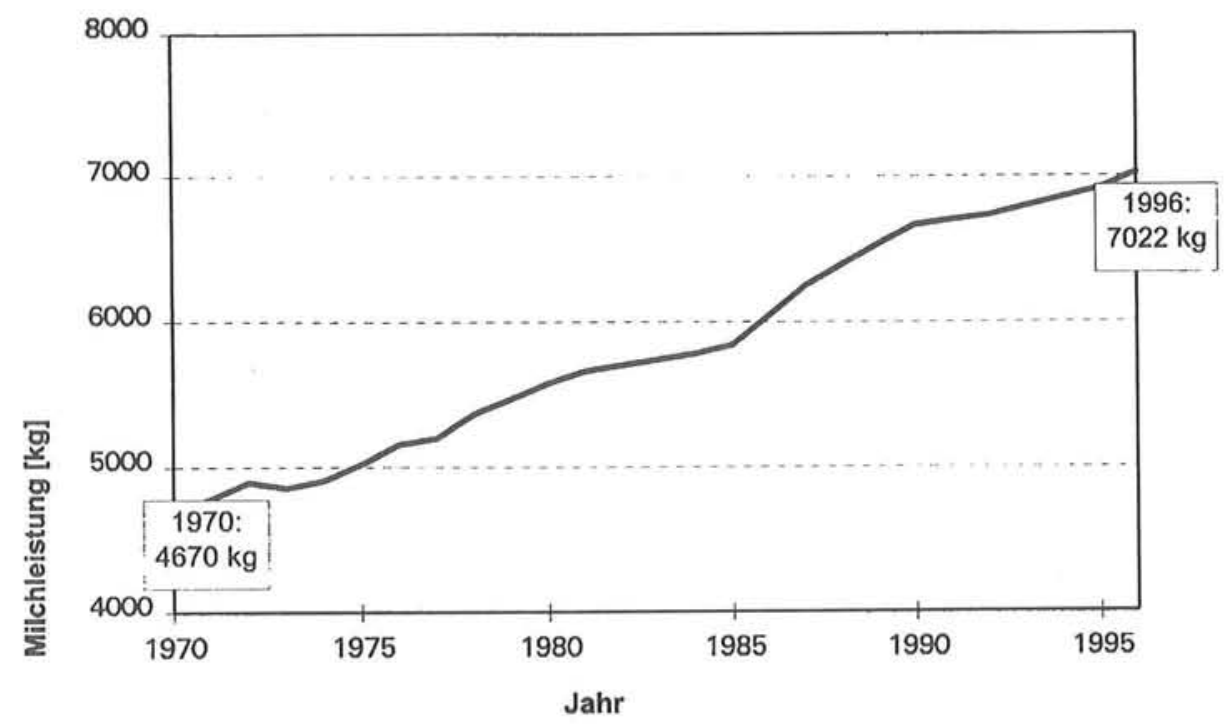

Abb. 3: Entwicklung der durchschnittlichen Milchmenge bei Schwarzbunten (MLP) in den alten Bundesländern (Quelle: ADR-Jahresberichte) (Development of average milk yield in tested Black-pied cattle of the old federal land (source: ADR-yearly reports))

Im gleichen Zeitraum gingen die sterilitätsbedingten Abgänge geringfügig zurück, während sich die krankheitsbedingten Ausfälle von 13,4\% auf 27,4\% um mehr als das Doppelte erhöhten (Abb. 4). Diese Entwicklung beruht wiederum vor allem auf einer überproportionalen Zunahme der Abgänge auf Grund von Euterkrankheiten (1970: 4,7\%, 1996: 15,0\% ). Unter Berücksichtigung der in Tabelle 1 aufgeführten Korrelationskoeffizienten besteht kaum ein Zweifel daran, daß die Entwicklung bei den krankheitsbedingten Abgängen ein vorwiegend genetisches Problem ist. Fast alle der auf diesem Gebiet durchgeführten populationsgenetischen Untersuchungen belegen, daß die einseitige Selektion auf hohe Milchmengenleistungen eine erhöhte Disposition ge- 


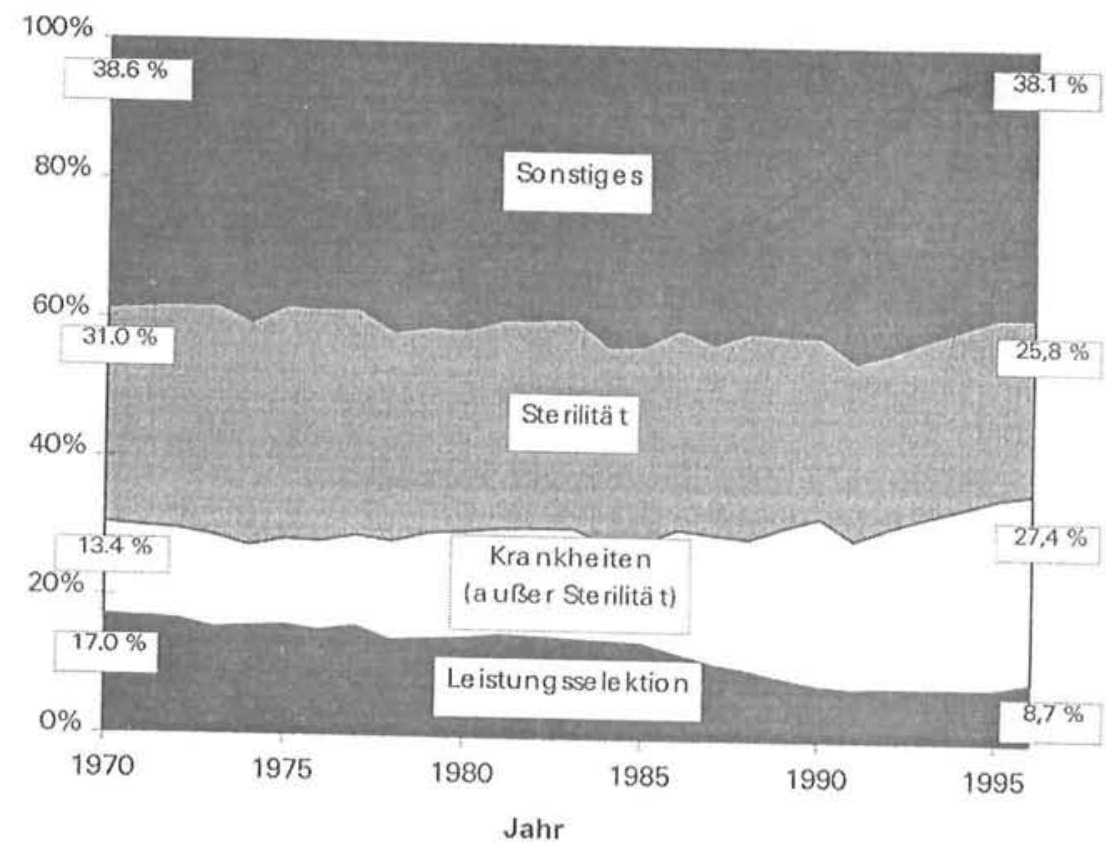

Abb. 4: Entwicklung der Kuhabgänge nach Ursachen bei Schwarzbunten (MLP) in den alten Bundesländern (Quelle: ADR-Jahresberichte) (Development of the cow losses in tested Black-pied cattle of the old federal land on base of various reasons (source: ADR-yearly reports))

genüber Mastitisinfektionen, Stoffwechselerkrankungen und Reproduktionsstörungen nach sich zieht. Wie die Entwicklung bei den sterilitätsbedingten Ausfällen zeigt, treten solche genetischen Zusammenhänge aber nicht immer auch phänotypisch in Erscheinung. So können z.B. verbesserte Technologien und Verfahren der Haltung, der Fütterung, der Tiergesundheits- und Fruchtbarkeitsüberwachung oder Sonderbehandlungen von leistungsstarken Kühen genetisch ungünstige Beziehungen zeitweise überlagern. Da aber die biologischen Adaptationssysteme nicht beliebig strapazierbar sind, werden auch die besten Verfahren der Umweltgestaltung das Auftreten biologischer Antagonismen dauerhaft nicht verhindern können. Die hierbei wirkenden genetischen Mechanismen wurden bereits beschrieben. Nachteilige Auswirkungen auf einzelne Fitneßkomponenten sind aber gleichzeitig auch Ausdruck von konkreten physiologischen Veränderungen. Beim Milchrind hat sich z.B. gezeigt, daß zwischen dem genetischen Leistungspotential während der Hochlaktation und dem genetischen Potential für Futteraufnahme eine erhebliche Diskrepanz bestehen kann (BRANDT et al., 1985; LEUTHOLD et al., 1991). Zwar wurden zwischen Milchmenge und Futteraufnahme ausnahmslos positive Beziehungen nachgewiesen, die genetischen Korrelationskoeffi- 
zienten rangieren jedoch mit $0,14 \ldots 0,89$ innerhalb eines sehr weiten Bereiches (HOOVEN et al., 1972; BRANDT et al., 1985; VAN ARENDONK et al., 1991), so daß der Zuchtfortschritt im Merkmal Futteraufnahme dem Zuchtfortschritt im Merkmal Milchleistung offenbar nicht in adäquater Weise folgen konnte. Dies induziert Belastungssituationen, die vor allem während der Hochlaktation schnell in pathophysiologische Zustände überwechseln können. Einige besonders wichtige Zusammenhänge lassen sich wie folgt charakterisieren:

Vermutlich gehen vom sich entwickelnden Kalb und den es umgebenden Geweben des Reproduktionssystems Primärsignale aus, die das Genom veranlassen, bereits mehrere Tage vor dem Partus tiefgreifende Veränderungen in den Stoffwechselabläufen einzuleiten. Dies äußert sich vor allem in einem Rückgang der Futteraufnahme (wahrscheinlich hauptsächlich durch steigende Östrogenwerte induziert), einem abgesenkten Insulin- und IGF-Spiegel sowie in erhöhten Konzentrationen der Hormone STH und Glukagon (GIESECKE, 1990; STAUFENBIEL et al., 1990; STANGASSINGER, 1990; KOLB und ELZE, 1995).

Eine solche hormonelle Konstellation bietet zwar kurzfristig günstige Voraussetzungen für die Milchsynthese, erhöht aber gleichzeitig auch die Wahrscheinlichkeit dafür, daß der Energie- und Substratbedarf des biologischen Systems über das Futter nicht mehr abgedeckt werden kann. Die entstehende Energielücke wird aus mobilisierter Körpersubstanz, vor allem aus Körperfett geschlossen (STAUFENBIEL et al., 1990; LEUTHOLD et al, 1991; KOLB und ELZE, 1995). Wenn unter diesen Bedingungen der Glukosebedarf (vor allem für die Milchzuckersynthese) größer als die Neubildungsrate ist, wird die hepatische Ketogenese forciert, was sich beim Wiederkäuer sehr ungünstig auf den Stoffwechsel von Nervenzellen auswirkt. Die Folge sind eine rückläufige Milchleistung und unterschiedlichste Störungen von biologischen Teilfunktionen.

Tabelle 1

Genetische Korrelationskoeffizienten zwischen Milchleistung und ausgewählten Fitneßkomponenten (Genetic correlations between milk yield and selected components of fitness)

\begin{tabular}{|c|c|c|}
\hline Stoffwechselkrankheiten & Euterkrankheiten & Reproduktionsmerkmale \\
\hline SIMIANER (1988) & FUNKE (1991) & DISTL (1990) Einsatzleistung/ \\
\hline M-kg/Ketose (ja/nein) & M-kg/verschiedene Mastitismerkmale & Verzögerungszeit \\
\hline \multirow[t]{3}{*}{0,65} & \multirow[t]{3}{*}{$0,12 \ldots 0,92$} & 0,59 \\
\hline & & Einsatzleistung/NR60 \\
\hline & & $-0,67$ \\
\hline URIBE et al. (1995) & DUDA und PIRCHNER (1989) & HOECKSTRA et al. \\
\hline M-kg/Ketose (ja/nein) & M-kg/Mastitis (ja/nein) & (1994) \\
\hline \multirow[t]{7}{*}{0,77} & \multirow[t]{2}{*}{0,70} & $\mathrm{M}-\mathrm{kg} / \mathrm{NR} 56$ \\
\hline & & $-0,26$ \\
\hline & SIMIANER (1988) & PÖSÖ et al. (1996) \\
\hline & M-kg/Mastitis (ja/nein) & M-kg/Ovulationsstörungen ( $\mathrm{ja} /$ nein) \\
\hline & 0,51 & 0,42 \\
\hline & & M-kg/Metritis (ja/nein) \\
\hline & & 0,68 \\
\hline
\end{tabular}


Die Belastungen, denen der Stoffwechsel während der Laktation ausgesetzt ist, lassen sich mit folgenden Zahlen charakterisieren:

Bei einer Milchleistung von $30 \mathrm{~kg} / \mathrm{d}$ ist mit einer Lipidmobilisation von 4,9 Mol Fettsäuren (ca. 1300g) und 1,7 Mol Glycerin (156g) zu rechnen, wobei die gleichzeitige Neuveresterung etwa 2,9 Mol Fettsäuren (ca. 780g) pro Tier und Tag beträgt (GIESECKE et al., 1987). Der Glukoseentzug aus dem Blut beträgt nach Angaben von GRAF (1984), PIATKOWSKI et al. (1990) und BLUM (1992) etwa $75 \mathrm{~g} / \mathrm{kg}$ Milch (50 $\ldots 100 \mathrm{~g}$ ) woraus sich bei gleicher Leistung ein Glukosebedarf von $2250 \mathrm{~g}$ plus $500-$ $600 \mathrm{~g}$ für Erhaltung (z.B. Nervenzellen und Erythrozyten) ableiten läßt. Die aufgeführten Beispiele lassen erkennen, daß Kühe mit hohen Milchmengenleistungen (= hohe Laktoseabgabe) / Einheit Futteraufnahme und mit hohen Lipolyseraten gegenüber Stoffwechselerkrankungen besonders gefährdet sind. Werden z.B. die beiden extremen Milchrassen Holstein Friesian (höchste Milchmengenleistung, hoher Glukosebedarf) und Jersey (höchste Milchinhaltsstoffe, geringer Glukosebedarf) hinsichtlich dieser Kriterien verglichen, so liegt der physiologische Vorteil eindeutig auf Seiten der Jerseys.

Tabelle 2

Ergebnisse eines Rassenvergleiches zwischen Jersey- und HF-Kühen (SCHMIDT, 1993) (Results of a breed comparison between Jersey- and Holstein Friesian-cows)

\begin{tabular}{|c|c|c|c|c|c|c|}
\hline Rasse (n) & $\begin{array}{c}\text { Körpermasse } \\
(\mathrm{kg}) \\
\text { und } \mathrm{KM}^{0,75}\left(^{*}\right)\end{array}$ & $\begin{array}{c}\text { Energie- } \\
\text { aufnahme } \\
\text { (MJ NEL/ } \\
\text { Kuh und d) }\end{array}$ & $\begin{array}{l}\text { Laktose- } \\
\text { menge } \\
(\mathrm{kg} / \mathrm{Kuh} \\
\text { und d) }\end{array}$ & $\begin{array}{c}\text { (A) } \\
\text { g Laktose-/ } \\
\text { MJ NEL }\end{array}$ & $\begin{array}{c}\text { (B) } \\
\text { g Laktose-/ } \\
\mathrm{kg} \mathrm{KM}^{0.75}\end{array}$ & $\begin{array}{c}\text { Acetonkon- } \\
\text { zentration in } \\
\text { der Milch } \\
(\mathrm{mmol} / \mathrm{l})\end{array}$ \\
\hline $\mathrm{HF}(16)$ & $543\left(112,5^{*}\right)$ & 117,55 & 1,091 & 9,70 & 9,28 & 66,1 \\
\hline Jersey (16) & $375\left(82,2^{*}\right)$ & 82,96 & 0,588 & 7,15 & 7,09 & 32,8 \\
\hline
\end{tabular}

Ihre relativ geringe Laktoseabgabe/Einheit Futteraufnahme hatte in Untersuchungen von SCHMIDT (1993) auch eine deutlich geringere Acetonbelastung zur Folge. Wie sich außerdem andeutet, sind die energetischen Zusammenhänge zwischen Futterenergieaufnahme und $\mathrm{KM}^{0,75}$, Futterenergieaufnahme und EKM-Leistung sowie zwischen $\mathrm{KG}^{0,75}$ und EKM-Leistung in beiden Rassen sehr ähnlich. Lediglich die unterschiedliche Verwendung des aufgenommenen Futters (für Inhaltsstoffe oder Milchmenge) führt schließlich auch zu Unterschieden in der Ketonkörperbelastung. Hinter solchen Befunden verbirgt sich vermutlich ein wesentlicher Grund für die hervorragenden Vitalitätseigenschaften, welche Jerseys gegenüber Rassen mit hohen Milchmengenleistungen auszeichnen.

Untersuchungen von ROBINSON et al. (1994) und GRAML et al. (1995) haben gezeigt, daß hohe STH-Spiegel und niedrige Insulinspiegel bei Besamungsbullen in positiver Beziehung $\mathrm{zu}$ deren Zuchtwerten für Milchmenge, Milchfettmenge und Milcheiweißmenge stehen. Vergleichbare Ergebnisse ergaben sich aus einer Arbeit von BONCZEK et al. (1988) an Milchkühen, in welcher Tiere mit einem hohen genetischen Leistungspotential ebenfalls höhere STH und niedrigere Insulinspiegel aufwiesen als weniger leistungsstarke Tiere. Auf ähnliche, leistungsassoziierte Auswirkun- 
gen verweist GRAF (1984) hinsichtlich der Schilddrüsenhormone. Nach dem derzeitigen Erkenntnisstand deutet vieles darauf hin, daß katabol orientierte Hormonkonstellationen:

- eine wesentliche Grundlage für hohe Mengenleistungen sind,

- nicht geschlechtsbegrenzt vererbt werden und

- während der Laktation die Basis für Stoffwechselimbalancen bereiten.

An dieser Stelle sei jedoch darauf verwiesen, daß der Wechsel zwischen kataboler und anaboler Stoffwechsellage einen tiefen physiologischen und zweifellos auch evolutionären Sinn ergibt. Zum einen ist das reproduktionsbedingte An- und Abschalten der Laktation fester Bestandteil des genetischen Fitnessprogramms, zum anderen sind biologische Puffersysteme für den Ausgleich von energetischen Mangelsituationen auch in Wildpopulationen von essentieller Bedeutung. Beim Milchrind wurden sie jedoch mit einer viel höheren Priorität weiterentwickelt, als weniger leistungsrelevante Mechanismen. Darüber hinaus postuliert GIESECKE (1990), daß mit der Leistungsselektion der Zeitpunkt des Laktationsmaximums immer dichter in Richtung Geburt verlagert wurde, so daß sich die verzehrshemmenden Einflüsse des Östrogenspiegels stärker bemerkbar machen konnten als in weniger ausgezüchteten Populationen.

Stoffwechselstörungen sind nicht nur in ihren primären Erscheinungsformen von Bedeutung, sondern sie erhöhen auch die Wahrscheinlichkeit für das Auftreten von Folgeerkrankungen. Obwohl die Untersuchungen zu den kausalen physiologischen Beziehungen zwischen Leistung, Stoffwechsel und Immunsystem noch ganz am Anfang stehen, kann es heute kaum noch einen Zweifel daran geben, daß von einem hohen Milchleistungspotential immunsuppressive Effekte auf die Individuen einer Population ausgehen (s. Tab. 1). In physiologisch orientierten Arbeiten von TARGOWSKI und KLUCINSKI (1983), TARGOWSKI et al. (1985), KLUCINSKI et al. (1988), ROPSTAD et al. (1989) und KANDEFER-SZERSCEN et al. (1992) zeigte sich, daß eine negative Energiebilanz bzw. eine ketotische Stoffwechsellage, wie sie bei Kühen mit hohen Leistungen häufig beobachtet werden, die Leukozytenzahl reduziert und deren immunologische Funktionalität beeinträchtigt. In eigenen Arbeiten (MÜLLER et al, 1997; MÜLLER et al., 1998) wurden mehrere immunkompetente Merkmale und Stoffwechselindikatoren an potentiellen Besamungsbullen mit unterschiedlichen Pedigree-Zuchtwerten verglichen. Hierbei zeichneten sich Bullen mit einem hohen Pedigreezuchtwert-Milch (P-RZM) von solchen mit einem niedrigeren P-RZM z.B. durch höhere Glukosespiegel und Erythrozytenzahlen aus, was als ein Hinweis auf günstigere physiologische Voraussetzungen für die Milchsynthese gewertet wurde. Gleichzeitig besaßen diese Tiere aber auch geringere Leukozytenzahlen, womit sie gegenüber der Vergleichsgruppe immunologisch benachteiligt waren. Weiterhin fiel auf, daß die Beziehungen zwischen einigen immunkompetenten Merkmalen und Stoffwechselindikatoren auch unter veränderten Bedingungen (Energiebilanz, Prüfzeitpunkt) relativ stabil blieben.

Die in Tabelle 1 aufgeführten Korrelationskoeffizienten signalisieren, daß sich mit dem genetischen Leistungsspotential auch die Wahrscheinlichkeit für das Auftreten von Reproduktionsstörungen erhöht. Wie bereits erwähnt, spielen die Lipolyserate 
(Energiebilanz), die Glucosebilanz und die Ketonkörperproduktion auch in der Pathogenese von Erkrankungen des Reproduktionssystems eine entscheidende Rolle. In einer Arbeit von BUTLER und SMITH (1989) wurde nachgewiesen, daß die Körpermassemobilisation p.p. (KMM) den Erstbesamungserfolg (EBE) maßgeblich mitbestimmt. Während in einer Gruppe mit geringer KMM der EBE bei $65 \%$ lag, erreichte die Gruppe mit mittlerer KMM nur 53\%. Besonders dramatisch war aber die Situation in der Gruppe mit hoher KMM. Hier wurden nur 17\% aller Tiere nach der ersten Besamung tragend. Eine unzureichende Glucoseverfügbarkeit beeinträchtigt zusätzlich noch den Stoffwechsel von Nervenzellen und damit auch den Reifungsprozeß von Tertiärfollikeln (KOLB und ELZE, 1995). Gleichzeitig sinken die Insulinkonzentration und der Gehalt an IGF-1, während die Cortikoliberin-, ACTH-, Glucocorticosteroidund STH-Werte ansteigen, wodurch die Freisetzung der für den normalen Ablauf des Reproduktionszyklus verantwortlichen Östrogene, von GnRH, FSH und von LH gehemmt wird (LOTTHAMMER, 1990; PLYM FORSHELL et al., 1991; KOLB und ELZE, 1995).

\section{Schlußfolgerung}

Die konsequente Erhöhung der Milchmengenleistung hat physiologisch nachvollziehbare Veränderungen fitneßrelevanter Merkmale zur Folge. Unter Berücksichtigung des beachtlichen, z.T. auch genetisch bedingten Leistungsanstieges, der während der letzten Jahrzehnte realisiert werden konnte, scheint es nunmehr erforderlich, allgemeine Merkmale und Milchinhaltsstoffe bei der Selektion stärker als bisher zu gewichten. Wie neuere Untersuchungen von MACK et al. (1997) zeigen, ist solch eine Forderung längst auch betriebswirtschaftlich begründbar. Unter den derzeitigen agrarpolitischen Rahmenbedingungen kann das nicht heißen, die Priorität der Leistungsselektion in Frage zu stellen, wohl aber ihre relative Bedeutung. Vom ethischen, wirtschaftlichen, physiologischen und nicht zuletzt auch vom verbraucherorientierten Standpunkt aus betrachtet sollte die Ganzheitlichkeit der züchterischen Arbeit stärker in den Vordergrund gerückt werden.

\section{Literatur}

ADR-Jahresberichte, 1970-1997

BLUM, J.W.:

Physiologische Grundlagen hoher Milchleistung beim Rind, Schweiz. Arch. Tierheilk. 134 (1992), 213. 228

BLUM, J.W.; KUNZ, P.:

Stoffwechselprofile: Veränderungen von Hormonen und Metaboliten im Zusammenhang mit der Milchleistung, Schweizerische. Landwirtschaftliche Monatshefte 58 (1980), 453-464

BONCZEK, R.R.; YOUNG,C.WHEATON, J.E.; MILLER, K.P.:

Responses of somatotropin, insulin, prolactin and thyroxine to selction for milk yield in Holstein. J. Dairy Sci., Champaign, Ill. 71 (1988), 2471-2479

BUTLER und SMITH

(zit. n. KOLB und ELZE, 1995) 
BRANDT, A.; PABST, K.; SCHULTE-COERNE, H.; GRAVERT, H.O.:

DISTL, O.:

Die Heritabilität der Futteraufnahme. Züchtungskunde, Stuttgart 57 (1985), 299-308

Zucht auf Fruchtbarkeitsmerkmale beim Rind, Fortschritte in der Tierzüchtung. Verlag Eugen Ulmer, Stuttgart, 1990

DUDA, J.; PIRCHNER, F.:

Schätzung genetischer Parameter für Merkmale der Mastitisanfälligkeit in Oberbayerischen Kuhherden. Züchtungskunde, Stuttgart 61 (1989), 334-346

FALCONER, D.S.:

FUNKE, U.

Einführung in die quantitative Genetik. Verlag Eugen Ulmer, Stuttgart, 1984

Genetische Analyse direkter Merkmale zur Selektion auf Verbesserung der Mastitisresistenz beim

GIESECKE, D. Schwarzbunten Milchrind. HU Berlin, Diss., 1991

Metabolische Leistungsgrenzen bei Milchkühen. Symposium Energie und Fettstoffwechsel, HU Berlin 1990, 3-17

GIESECKE, D.; MEYER, J.; GRAF, F.; KOSAK, F.:

Stoffwechselbelastung, freie Fettsäuren und Ketogenese bei Kühen mit hoher Milchleistung, Fortschritte in der Tierphys. und Tierern. 18 (1987), 10-17

GRAF, F.:

Stoffwechsel und Endokrinologie von Hochleistungskühen. Züchtungskunde, Stuttgart 56 (1984), 344350

GRAML, R.; OLBRICH-BLUDAU, A.; SCHWAB, M.; SCHALLENBERGER, E.; SCHAMS, D.; PIRCHNER, F.:

Relationship between plasma hormone and metabolite levels and breeding values of bulls, J. Anim. Breed. Genet. 112 (1995), 313-326

HOECKSTRA, J.; VAN DER LUGT, A.W.; VAN DER WERF, J.H.J.; OUWELTJES, W.:

Genetic and phenotypic parameters for milk production and fertility traits in upgraded dairy cattle. Livestock Pro. Sci., Amsterdam 40 (1994), 225-232

HOOVEN, N.W. JR.; MILLER, R.H.; PLOWMAN, R.D.:

Relationship among whole-and part-lactation gross feed efficiency, feed consumption, and milk traits, J. Dairy Sci., Amsterdam 55 (1972), 1113-1122

KANDEFER-SZERSZEN, M.; FILAR, J.; SZUSTER-CIESIELSKA, A.; RZESKI, W.:

Suppression of interferon response of bovine leukocytes during clinical and subclinical ketosis in lactating cows, Deutsche Tierärztliche Wochenschrift, 99 (1992), 440-443

KLUCINSKI, W., MIERNIK-DEGORSKA, E., DEGORSKI, A., TARGOWSKI, S., WINNICKA, A.: Effect of Ketone Bodies on the Mitogenic Response of Bovine Milk Lymphocytes. J.Vet.Med.A., 35 (1988), 626-631

KOLB, E.; ELZE, K.:

Durch Energiemangel beim Rind ausgelöste Fortpflanzungsstönungen. Der praktische Tierarzt, 1995, 623-626

LEUTHOLD, G.

Aspekte der Nutzanwendung von Erkenntnissen der biochemisch-physiologischen Genetik in der Tierzüchtung. Arch. Tierz., Berlin 15 (1972), 143-161

LEUTHOLD, G.; WILHELM, H.; MÚLLER, U.:

Heritabilität und Zuchtwertdifferenzen der Futteraufnahme und Energiebilanz von Kühen des Schwarzbunten Rindes, Züchtungskunde, Stuttgart 63 (1991), 342-351

LOTTHAMMER, K.H.:

Beziehungen zwischen einigen Blut- und Milchinhaltsstoffen als Indikatoren der Energieversorgung und der Fruchtbarkeit sowie Euter- und Stoffwechselstörungen bei Milchrindern. Symposiumsbericht, Energie- und Fettstoffwechsel der Milchkuh, HU Berlin, 1990, 162-174

MACK, G.; WEIDELE, A.; DISTL, O.; ZEDDIES, J.:

Grenznutzen der Leistungsverbesserung in der Milchproduktion. Züchtungskunde, Stuttgart 69 (1997), $322-333$ 
MIN, S.H.; McCUTCHEON, S.N.; MACKENZIE, D.D.S., WICKHAM, B.W.:

Plasmametabolite and hormone concentrations in Friesian calves of low or high genetic merit: effects of sex and age, Anim.Prod., Edinburgh 56 (1993), 17-27

MÜLLER, U.; LEUTHOLD, G.; REINECKE, P.:

Möglichkeiten einer indirekten Selektion gegen residual feed intake beim Milchrind. Arch. Tierz., Dummerstorf 38 (1995), 277-287

MÜLLER, U.; LEUTHOLD, G.; DALLE, T.; REINECKE, P.:

Der Einfluß des genetischen Milchleistungspotentials auf immunkompetente Merkmale und Stoffwechselindikatoren bei gefütterten und hungerbelasteten Milchrindbullen, Arch. Tierz., Dummerstorf 40 (1997), 493-507

MÜLlER U., DALLE, T.; LEUTHOLD, G.; STAUFENBIEL, R.; KRÜGER, M.; KRETZSCHMAR, C.; SEIDLER, T.; SCHMIDT, M.F.G.: Züchtungsbiologische Charakterisierung der Beziehungen zwischen Stoffwechselindikatoren und immunkompetenten Merkmalen bei hungerbelasteten Milchrindbullen, Arch. Tierz., Dummerstorf 41 (1998) 359-370

PIATKOWSKI, B.; GURTLER, H.; VOIGT, J.:

Grundzüge der Wiederkäuerernährung. Gustav Fischer Verlag Jena, 1990

PIRCHNER, F.:

Genetischer Antagonismus bei Rindern. Züchtungskunde, Stuttgart 51 (1979), 423-433

PLYM FORSHELL, K.; ANDERSON, L.; PEHRSON, B.:

The relationships between the Fertility of Dairy Cows and Clinical and Biochemical Measurements, with special reference to Plasma Glucose and Milk Acetone, J.Vet.Med. A 38 (1991), 608-616

PÖSÖ, J.; MÄNTYSAARI, E.A.:

Genetic relationships between reproductive disorders, operational days open and milk yield. Livestock Prod. Sci., Amsterdam 46 (1996), 41-48

ROBINSON, D.L.; HAMMOND, K.; MCDOWELL, G.H.:

Relationships between breeding values and physiological responses to fasting and refeeding in dairy bulls: update for young animals. J. Anim. Breed. Genet. 111 (1994), 257-264

ROPSTAD, E.; LARSEN, H.J.; REFSDAL, A.O.: Immune Function in Dairy Cows Related to Energy Balance and Metabolic Status in Early Lactation, Acta veterinaria Scandinavica, 30 (1989), 209-219

SCHMIDT, E.:

Untersuchungen zu Einflußfaktoren auf Futteraufnahme und Energieverwertung - Eine experimentelle

SIMIANER, H.: Studie an Holstein Friesian, Jersey und Schwarzbuntem Milchrind. HU Berlin, Diss., 1993

Zusammenhänge zwischen Krankheitsanfälligkeit und Milchleistungsmerkmalen beim Norwegischen Rotvieh. Dsch. tierärztl. Wschr. 95 (1988), 137-192

STANGASSINGER, M:

Über die regulatorische Funktion der Pankreas-Hormone des Milchrindes bei Belastungszuständen. Symposium Energie- und Fettstoffwechsel, HU Berlin (1990) M, 429-439

STAUFENBIEL, R.; ROSSOW, N.; STAUFENBIEL, B.; BAUER, J.:

Untersuchungsgrößen des Energie- und Fettstoffwechsels, Symposium Energie- und Fettstoffwechsel. HU Berlin (1990), 26-99

TARGOWSKI, S.; KLUCINSKI, W:

Reduction in mitogenic response of bovine lymphocytes by ketone bodies. Am. J. Vet. Res. 44 (1983), 828-830

TARGOWSKI, S.; KLUCINSKI, W,; LITTLEDIKE, E.T.; HOY, D.E.:

Suppression of mitogenic response of bovine lymphocytes during experimental ketosis in calves. Am. J. Vet. Res. 46 (1985), 1378-1380

URIBE, H.A.; KENNEDY, B.W.; MARTIN, S.W.; KELTON, D.F.:

Genetic parameters for common health disorders of Holstein Cows. J. Dairy Sci., Champaign, Ill. 78 (1995); 421-430

VAN ARENDONK, NIEUWHOF, G.J.; VOS, H.; KORVER, S.:

Genetic aspects of feed intake and efficiency in lactating dairy heifers, Livestock Prod. Sci., Amsterdam 29 (1991), 263-275 
WOOLLIAMS, J.A.; LOVENDAHL, P.:

Physiological attributes of male and juvenile cattle differing in genetic merit for milk yield: a review. Livestock Prod. Sci., Amsterdam 29 (1991), 1-16

Eingegangen: 14.10 .1998

Akzeptiert: 01.12 .1998

Anschriften der Verfasser

Dr. UWE MÜLLER, PD Dr. habil. PETER REINECKE,

PD Dr, habil. WOLFGANG LEUCHT

Humboldt-Universität zu Berlin

Landwirtschaftlich-Gärtnerische Fakultät

Institut für Nutztierwissenschaften

FG Züchtungsbiologie und molekulare Tierzüchtung

Philippstr. 13

D-10115 Berlin

Diplom-Agraringenieur TORSTEN DALLE

RBB Rinderproduktion Berlin-Brandenburg $\mathrm{GmbH}$

Mielestr. 2

D-14542 Werder (Havel) 